\title{
INHALED RADIOACTIVE PARTICLES AND GASES
}

T HE third Hanford Biology Symposium, held at Richland, Washington, U.S.A., during May 4-6, 1964, was devoted to inhaled radioactive particles and gases. It was organized by a committee of Hanford scientists under the chairmanship of Dr. W. J. Bair. The 200 participants included representatives of nine countries, who were welcomed by Dr. H. A. Kornberg, head of the Hanford Biology Laboratories.

Proceedings were opened by Dr. W. E. Lotz, head of the Division of Biology and Medicine of the U.S. Atomic Energy Commission. Consequent on the test ban treaty a proliferation of effort was in progress, and a glimpse was given of the novel and interesting outlets for atomic expertise which were being attempted.

Foremost among these were the systems for nuclear auxiliary power units; these are heat sources, operated by various nuclides, in the range $40-240$ kilocuries, which generate electricity by thermoelectric inversion, using semiconductors. Lights for marine buoys with a life of 10 years produce $10-60 \mathrm{~W}$; a $25-\mathrm{W}$ generator for a navigational satellite was also described.

Nuclear-powered rocket engines carry a finite risk of re-entry after launching which can be faced by designing either for burn-up or by ensuring that the isotope container remains intact. At a height exceeding 100,000 ft. a fuel cone can disintegrate or burn-up into particles smaller than $10 \mu$ which dilute sufficiently in the biosphere to offer no risk; a ground contamination of $2,000 \mathrm{mc}$. $/ \mathrm{mile}^{2}$ would not be exceeded.

Dr. T. T. Mercer (Lovelace Foundation, New Mexico) made $a_{\text {c }}$ comparison of laboratory and field problems in handling aerosols of mixed fission products; there were hezards associated with natural sources of radon, with fuel melting in accidents and with fuel recovery operations.

Generation of laboratory clouds by aqueous sprays presented the possibility of the aerosol behaving anomalously owing to the particles carrying electric charges. These could arise from statistical fluctuations of ion concentration when liquid was fragmented from bulk. Natanson's theory had been verified at low numbers of particles, the mean charge being proportional to the $3 / 2$ power of the diameter and the square root of the number per $\mathrm{cm}^{3}$. The charge could amount to 100 electrons per particle. At higher concentrations the charge was independent of the number and proportional to the square root of the diameter.

Such charges can seriously distort deposition from laboratory aerosols and a tritium source was used to discharge them. Sampling had been most successfully carried out with the conifuge, which is a true aerosol spectrometer, in contrast with other so-called instruments, because the aerosol intake stream is narrow and confined between guard layers of pure air, which ensures that the site of doposition is sharply determined by Stokes's diameter. Mass parameters are needed for isotope inhalation investigations and their derivation from particle counts involves shape and density complications. The cascade impactor is useful for sampling because it yields mass parameters directly. Small jets, $0.015 \mathrm{~cm}$ diameter operating at 1/3 sonic velocity, had been useful for impacting very small particles.

Particles, particularly those containing plutonium which constituted an inhalation hazard when airborne, were discussed by K. Stewart (U.K. Atomic Energy Authority, Aldermaston). One problem was the large amount of extraneous material which accompanied the dangerous aerosol. For example, $4 \times 10^{-5} \mu \mathrm{c} . / \mathrm{m}^{3}$ of plutonium was equivalent to only 100 particles $/ \mathrm{m}^{3}$ of $1 \mu$ diameter; this is only a minute fraction of the total adventitious aerosol in many circumstances.

High-altitude burn-up results in small particles because the entire mass is expanding; near the surface of the Earth there is massive and inhomogeneous entrainment. There are great difficulties in predicting the outcome o a quenched fireball in water.

Oxidation processes result in aerosols by break-away of particles of oxide from the surface; a higher temperature gives coarser particles. Some alloys had an adherent oxide film. In an ignition of plutonium only some $0 \cdot 1$ per cent of the material was likely to be found in the hazardous range of particle size. Whereas uranium aerosols contained large aggregates of spheres, particles of plutonium were usually individual fragments.

Investigations of the particle size distribution of airborne plutonium in work rooms, using filtors, were described by B. V. Andersen, of the Hanford Laboratories. Membrane filters with $2 \mu$ pore size had been found useful and 5-50 $\alpha$-tracks per particle were obtained by autoradiography. As a rough rule, doubling the particle size resulted in a ten-fold increase in radioactivity. ${ }^{239} \mathrm{PuO}_{2}$ was found in lognormal distributions with geometrical standard deviation $\left(\sigma_{g}\right)$ of $1 \cdot 8-3.85$ per cent of the activity was in the largest 1 per cent of sizes. Plutonium carbide melt operations yielded the finer clouds with a maximum diameter of $1 \cdot 7 \mu$, GMD $0 \cdot 2-0 \cdot 48 \mu$ and $g=$ $1 \cdot 4-1 \cdot 8$.

R. E. Albert et al. (Department of Industrial Medicine, New York University) have developed techniques for making monodisperse spheres of 'Lucite' and of iron oxide using a spinning disk. The sizes are uniform within 10 per cent standard deviation over a range of $1-8 \mu$. The lucite solution which is fed to the disk contains a tagging isotope-either in carrier-free form or chelated with acetyl acetone. The presence of acetyl acetone in the xylene solution was found essential to the production of spheres with unwrinkled surfaces. These particles are not taken up by phagocytes. Good uniformity has also been achieved in the dispersal of sols of colloidal iron oxide. These particles are stable in water and are taken up by phagocytes. Various isotopes have beon used for examining the rate of leaching out. In each case the particles require to be collected from the spinning disk generator and redispersed for experimental work.

Montmorillonite clay will absorb a number of radionuclides by ion exchange and can then be dispersed as an aerosol by spraying a suspension. The technique was described by S. Posner (Lovelace Foundation, Albuquerque). He also found that latex spheres would absorb iodine-13I from solution in ethyl alcohol. Data on isotope leach-out were given.

Two papers dealt with radioactive dust and gas from uranium mines. H. Fusamura et al. had sampled radon in Japanese mines with an ionization chamber and transistorized vibrating reed electrometer. The concentration ranged from 0.3 to $10.0 \times 10^{-7} \mu c . / c . c$. and increased to $3 \times 10^{-6} \mu c$./c.c. when the mine ventilation was stopped. Daughter products were collected by filtration and the free ion concentration roughly estimated by placing a $2-\mathrm{m}$ long diffusion tube in front of the filter; it was reckoned that free ions accounted for 20 per cent of the RaA. The dust on the filter contained $0.01-0.08$ per cent $\mathrm{U}_{3} \mathrm{O}_{8}$ which corresponds to $1-45 \times 10^{-3} \mu$ c./c.c.

Dr. Palmer (Hanford Biology Laboratories) had taken a whole-body counter to two Colorado uranium mines, 
one of which was disused and one in operation. Sampling was performed with membrane filters for daughter products and activated charcoal for radon. The counter was used very soon after exposure and the entire length of the subject's body could be scanned. Some 20 per cent of activity was found in the head, 10 per cent in the neck and 70 per cent in the chest. The maximum chest concentration was in the right side, 3 in. off centre. It seemed that all the activity inhaled by the subjects while in the mines was still in their bodies when measured by the counter.

Several papers dealt with the elimination of dust from the lungs, including an outline of the processes by S. Engel. The theory of fluid transport, rather than carriage by phagocytes, to the pulmonary lymph nodes was supported by P. Gross, who considered that insoluble particles which deposit in the alveoli are sequestered on the wall in nodules or plaques, although a few penetrate the wall directly. Sequestered particles may be freed in due course, and eliminated vis the bronchial epithelium.

Three phases of clearance, at decreasing rates, were identified by L. J. Casarett and P. F. Milly (University of Rochester); these comprised bronchiolar clearance, phagocytosis from alveoli and transport to the lymphatics. The half-time for alveolar clearance ranges from 15 to 50 days and depends on the material and the animal. Much of the material deposited in the alveolar regions is found in macrophages after $2 \mathrm{~h}$. Chemitaxis is a factor, but different substances do not have half-times in line with their solubilities. Protein binding favours phagocytosis. It was suggested, in the discussion on this paper, that the phagocytosis of radioactive particles may be delayed.

In formulating a theory of dust clearance account had to be taken of the long period during which some particles remained in the alveoli; a macrophage development sequence was visualized in which cells of the alveolar epithelium, which may have been penetrated by insoluble particles. periodically detach themselves. According to the way in which the cell lies in the alveolar wall, detachmont may proceed either into the air space or into the interstitium.

R. G. Thomas (Lovelace Foundation, Albuquerque) described experiments with aerosols of thorium, plutonium, niobium, uranium and polonium which were inhaled by dogs. All the data were grouped along a straight line when the proportion of lung content in the lymph nodes was plotted against time, using logarithmic scales. The proportion of the whole-body content eliminated in the fæces fell off exponentially much more rapidly than urinary excretion; this indicates the change-over from lung elimination as ciliary removal declines.

A biochemical examination of rabbit lung by $J$. M. Felts (University of California Medical Center) showed that 8-10 per cent of the oxygen uptake was used in lung tissue for the oxidation of long-chain fatty acids bound to serum albumin. Using carbon-14 it was shown that lung, in contrast to liver, converted fatty acids into phospholipids, mainly lecithin. Glucose probably acts as an acceptor intermediate. Lung phospholipids are a key component of the surfactant which stabilizes the alveoli by reducing surface tension to an extremely small value.

B. B. Boecker et al. (Lovelace Foundation) described a new whole-body plethysmograph in which a dog could be placed with its nose protruding through a rubber seal into an aerosol chamber. Air moving to and from the plethysmograph box was recorded with a pneumotachygraph inserted between the box and another sealed chamber. This system eliminates difficulties due to drift of pressure. The boxes were made of $\frac{3}{8}-$ in. 'Plexiglass' which responded to prossure changes in the room when strong winds blew outside.

An inhalation apparatus for small animals breathing ${ }^{14} \mathrm{CO}_{2}$ was described by D. L. Joftes (Now England Deaconess Hospital, Boston). The increase in carbon-14 in the atmosphere is important since carbon dioxide may be a direct precursor of DNA. Assuming the animal fixes 0.5 per cent of the carbon dioxide available, a dose of $0 \cdot 1$ rad was calculated in 10 days. The exposure chamber was provided with constant environment and a metered supply of carbon dioxide up to 4 per cent. Estimates of individual testes by autoradiography and liquid scintillation counting were planned.

Experiments on the inhalation of cæsium-137 by rats had been performed at the Lovelace Foundation by Miss R. Lie. Aerosols of the chloride and nitrate in concentrations between 0.05 and 9.0 per cent were used and tissue and excreta samples analysed for 4 months.

C. E. Miller (Argonne National Laboratory) gave an account of the examination of two persons who had accidentally inhaled cæsium-137. Whole-body analysis was performed by the tilting chair technique and the distribution of cæsium-137 and potassium-40 was found in a low background room using a simulated 7-crystal array. No appreciable amount of cæsium-137 was found in the lungs after 9 days from exposure. There was no translocation over 185 days and a biological half-time of about 80 days was indicated. The nuclide was distributed. throughout the body.

A case of human exposure to plutonium-239 was discussed by W. S. Snyder (Oak Ridge). On the model of the 'standard man', an elimination half-time of 120 days is assumed for insoluble lung deposits, most of this material being disposed of in the fæces. There is, however, some indication that plutonium-239, and other heavy metals, reach the blood in significant quantities, so that transfer to bone is possible. The bone may thus be the critical organ. Whereas the lung may carry a higher concentration for the first few years of exposure the bone content would eventually predominate and might become the limiting factor in permitting continuous exposure for a working life.

An investigation of the metabolism of radio-mercury by rats during chronic exposure for two months was performed by A. Rothstein and A. Hayes (University of Rochester). The body burden was determined by wholebody counting and fluctuated with the exposure régime. After three weeks, intake and excretion were in balance. On terminating exposure the body burden declined exponentially with a half-time of 15 days. Most mercury was found in the kidneys; there was an appreciable loss of mercury vapour via the lungs and the skin.

The production of test aerosols for lung clearance investigations in rabbits was the subject of a paper by A. Kajland et al. (Karolinska Institute, Stockholm). Octadecanol was dispersed by a spray-fed thermal generator with the nucleating substance dissolved in the octadecanol. The coefficient of variation in particle size was 5-10 per cent for diameters of $2 \mu-10 \mu$ and 20 per cent for $1 \mu-2 \mu$. A spinning disk spray gave a variation coefficient of 10 per cent for spheres of 'Lucite' produced from a 0.2 per cent solution in 5 per cent acetone and 95 per cent acetamide. The use of a mixed solvent is necessary if smooth, unwrinkled spherical particles are to be obtained.

Lung clearance was measured by a profile-scanning method which allowed multiple examinations and $r e$ exposure of a single animal. It had been observed that the phagocytosis of octadecanol was low.

A novel technique for measuring ciliary clearance from the lungs of rats was devised by A. A. Spritzer and J. A. Watson (Graduate School of Public Health, Pittsburgh). All material swallowed is collected through a polyethylene tube placed in the animal's cesophagus; the caudal end of the tube empties into a 100-c.c. bottle fastened to the flank. Rats wero kept alive for 7 days and daily collections were made; there was no intorference with swallowing. The pulmonary phlegm was flushed out by the water drunk. Following intratracheal injection of ${ }^{133} \mathrm{BaSO}_{4}$ particles it was found that 8 per cent, or about 
half the total pulmonary clearance, was by the ciliary mechanism.

Experiments on inhalation by man of aerosol particles were described by L. Dautrebande (Brussels) and W. Walkenhorst (Bergbauberufsgenossenschaft, Bochum, Western Germany); few particles were found in exhaled alveolar air, this being claimed to represent 90 per cent deposition. In contrast, C. N. Davies (Medical Research Council and London School of Hygiene and Tropical Medicine) emphasized, by comparing size distributions of lung dust and airborne dusts, that the access of particles to the alveoli of man during normal breathing, was more limited than was commonly supposed. Curves of alveolar deposition, derived by indirect arguments from total deposition experiments, considerably over-estimated alveolar deposition and could not bo explained by phagocytosis from the alveoli.

Calculation of the cancer-related dose from the inhalation of radon and daughter products had been carried out by a synthetic approach by B. Altshuler et al. (Institute of Industrial Medicine, New York University). The dose was estimated from the surface activity on the bronchial mucosa, not from material lodgod within pulmonary tissuo. The atmosphere was supposed to contain 100 pc. (picocuries) of radon, 150 unattached daughter atoms and $200 \mathrm{pc}$. total daughter products per litre. The deposition of ions, nuclei and particles in the different regions of the respiratory tract was calculated on Landahl's model and the mucus transit time worked out for each region; the available $\alpha$-activity on the surface of the bronchial mueus at all regions could thus be estimated. Allowing for the thickness of the mucus film and of the epithelial cells, an estimate was made of the dose to the basal cells of the bronchial opithelium throughout the regions. The thickness is a critical factor in the calculations. The basal cells of the segmental bronchi were found to receive the greatest dose, which amounted to $30 \mathrm{rads} /$ working year of $2,000 \mathrm{~h}$. In terms of animal experiments this indicates that the atmosphere assumed would deliver a carcinogenic dose in a working life-time.

A somewhat similar calculation was presented by W. Jacobi (Hahn-Meitner Institute of Nuclear Physics, West Berlin). He took $10^{4}$ particles $/ \mathrm{cm}^{3}$ with an average diameter of $70 \mathrm{~m} \mu$ for RaB and RaC and $100 \mathrm{~m} \mu$ for ThB and ThC. The mean percentages of unattached atoms are 100 per cent ThA, 25 per cent RaA. and 1 per cent RaB. Deposition and mucus transport in the various regions of the respiratory tract were ostimated and the mean biological half-time in the lungs was measured as $8 \mathrm{~h}$. $\mathrm{He}_{\mathrm{e}}$ found, like A.ltshuler, that the dose from inhaled decay products was not negligible and that the basal cells of the lower bronchi received the highost rate. His overall figure was about 30 per cent below that of Altshuler. It is evident that current normal exposure runs near to tho limit.

Observations on the contamination of Yugoslav miners of uranium were described by D. Djuric et al. (Institute of Occupational Health, Belgrade). Levels of radon up to 16 times the maximum allowable concentration had been recorded in the past, but improvement of the ventilation system had now been effected.

Assessment of polonium-210 in the urine correlated with radon exposure; the exposure to polonium- 210 from dust was gauged by determining polonium, radium and uranium in the dust. There is an exposure to polonium-210 from eigaretto smoke.

The pulmonary function of uranium miners had beon measured by V. E. A.rcher et al. (U.S. Public Health Service, Salt Lake City). The data suggest that working for short periods in high levels of radon and daughter products improves ventilatory function, but long exposures are detrimental above the losses resulting from ageing and from cigarette smoking. The effects of silicious dust and of radiation could not bo separated. The action of the latter comprises specific injury to the bronchial epithelium and diffuse injury to lung tissue. It has been suggested that the inhalation of positive ions causes the bronchi to contract while negative ions produce relaxation. A negative charge on dust particles has been claimed to increase phagocytosis.

G. Saccomanno (St. Mary's Hospital, Grand Junction, Colorado) reported an increase in the lung cancer of uranium minors. Most tumours were undifferentiated, in contrast to 71 por cent epidermoid for a non-uranium miner group. 79 per cent of the tumours were of the oat cell type with a preference for the hilar area.

A general reviow of the biological effects of radiation on the lung was made by M. Kuschner and N. Nelson (Now York University). The syndrome of radiation pneumonitis was defined in 1920. Bronchial mucosal changes include the evolution of abnormal epithelial cells which are sloughed off; along with this an interstitial fibrosis develops. The dose response relationship is poor. Effects have been reported at $500 \mathrm{r}$ and there is $20-50$ per cent incidence at 2,000-6,000 r. The fibrosis is related to deposits of inhaled activity; alveolar capillary block may follow with a reduction of diffusing surface area.

Cancer is bronchogenic in origin. Long exposure is necessary to induce it and it was first domonstrated experimentally in 1957. $10^{3}-10^{4}$ rads will induce tumours. $63 \mu \mathrm{c}$. of strontium -90 in a capsule will yield a squamous cell carcinoma of the lung when embedded for 3-15 months.

In calculating dose it is necessary to define a target area in an organ, because cancer does not begin in an organ but in a tissue. It is possible to over-interpret early metaplastic response; for example, the implantation of a 5.uc. ruthenium pellet produced denudation of epithelium followed by regeneration one or two cells deep; chemical irritants give a similar epithelial response. If a tumour is to appear it does so explosively after a steady induction period of metaplasia lasting, in rats, some 120 days; this follows the initial hyperplasia which increases for the first month. Work with S. Laskin showed that the tumour yield was proportional to the amount of radioisotope.

Peripheral tumours develop in areas of fibrosis which may play a part, as in scar cancer. Many Colorado miners have silicosis. Questioned on the nature of malignant change, the speaker said he regarded virus and radiation as co-carcinogens, each a tool which manipulated genetic material.

H. Cember (University of Cincinnati) had induced lung tumours in rats with the chloride and fluoride of cerium144. Intratracheal doses of $0 \cdot 5-50 \mu \mathrm{c}$. were injected. The mean radiation dose absorbed during survival time was calculated from lung clearance data and when plotted against the cumulative tumour frequency, extrapolated to zero at a value of $250 \mathrm{rad}$. This is interpreted as the threshold dose for lung tumour induction in rats by radioactive particles in the lungs.

The inhalation of ${ }^{144} \mathrm{CeO}_{2}$ by dogs was the subject of a paper by B. O. Stuart, A. W. Casey and W. J. Bair (G. E. Hanford Laboratories). Aerosols of various particle sizes between $0.09 \mu$ and $1.40 \mu$ were administered and the amount deposited ranged from 50 to $2,200 \mu \mathrm{c}$. The effective half-life was similar to the radioactive one, 285 days, and the biological half-time was $1.5-5$ years. Up to $900 \mu \mathrm{c}$. failed to produce significant change in blood gas or serum enzymo, but heavy doses up to 130 kilorads produced severe damage in lungs, mediastinal lymph nodes and liver. 22 per cent of the body burden at death was in the lungs, 44 per cent in the liver and 27 per cent in bone.

From the same laboratories a paper by J. W. Park, W. J. Clarke and W. J. Bair dealt with the chronic effects of inhaled ${ }^{239} \mathrm{PuO}_{2}$ in dogs. The aerosol was generated by calcining in air at $300^{\circ} \mathrm{C}$. The radiation dose to the lungs was 9-23 kilorads; the clinical symptoms were cardiopulmonary defect and lymphopenia. Bronchial and inter- 
stitial tumours were found, locally invasive and of multiple origin. No metastases wore seen. For the poriod of exposure, up to 48 months, the lung was the critical organ causing death.

An original technique for the safe radiological visualization of the human lung was due to G. V. Taplin et al. (University of California).

Serum radioalbumin in a 1 per cent solution is readily aggregated by adjustment of $p \mathrm{H}$ and heating at $79^{\circ}$ for $20 \mathrm{~min}$. 10-20 $\mathrm{m} \mu$ particles act like bacteria and are removed by the liver on first passage. By lowering the $p \mathrm{H}$ macro aggregates of 5-50 $\mu$ are formed. These are too large to pass through the capillaries of the lung, where they are temporarily trapped for $\mathrm{l}-2 \mathrm{~h}$. The lung can be scanned photographically during this period, and on the resulting picture it is possible to detect pulmonary emboli, lung infarets, pneumonia, atelectasis, certain tumours and cysts causing local ischæmia.

Work at the U.S. Public Health Service (Taft Laboratories, Cincinnati) on the intake of radiostrontium by infants was presented by B. Kahn et al. Levels in air, water and prepackaged baby foods were measured and the ingestion calculated. Retention was deduced by subtracting the activity in the excreta; strontium-89 and -90 are present in air in soluble forms.
The last papers read dealt with the removal of inhaled radioactive materials. I. Schmidtke (University Clinic, Freiburg-im-Breisgau) treated guinea pigs with diethylene triamine penta acetic acid after exposure to ${ }^{91} \mathrm{YCl}_{3}$ aerosol. A 95 per cent diminution of retention was found, in comparison with untreated animals, after 8 days. The action was mainly on the kidneys, and most of the activity was recovered from the urine. E. G. Tombropolous (Hanford Laboratories) considered that cholating agents were the most effective means, when applicable, of removing radioactive particles from the lungs. Drugs which decrease mucus secretion or increase phagocytosis are also worth testing. F. Gensicke and H. W. Nitschke (German Academy of Sciences, Berlin-Buch, G.D.R.) reported that sodium hexametaphosphate had a scavenging effect on inhaled yttrium-91 in mice.

Papers read in title dealt with a spinning disk atomizer, a routine technique for estimating plutonium in large biological specimens, fall-out from burnt-up uranium and plutonium, $\mathrm{Zr}-{ }^{95} \mathrm{Nb}$ in human lungs from medium life fission products in the atmosphere, atmospheric analysis for total B-activity and the monitoring of human subjects who had accidentally inhaled fission products.

The papers will be published in full in the December 1964 issue of Health Physics.

C. N. Davies

\section{BIOSYNTHETIC PATHWAYS IN HIGHER PLANTS}

$\mathrm{T}$ HE biosynthetic pathways leading to all the major groups of compounds present in higher plant tissues were discussed at the seventh annual general meeting of the Plant Phenolics Group which was held at Bodington Hall, University of Leeds, during April 13-15. The wide range of topics covered by the symposium coincided with a decision by members to change the name of the Group to the Phytochemical Group with the view of promoting the advancement of the chemistry, biochemistry and related sciences of all plant products.

The opening address was given by Prof. N. A. Burges (University of Liverpool), who discussed the biological aspects of biochemical reactions and the possible differences between reactions in vivo and in vitro. This is an important consideration which is still sometimes overlooked by chemists. Biochemical reactions in vivo are commonly studied by feeding in potential precursors labelled with 'heavy' or radioactive isotopes. Dr. T. Swain (Low Temperature Research Station, Cambridge) gave a critical account of these methods. In particular he noted that feeding experiments often led to an understanding of the starting and end points of a pathway but gave little information regarding tho individual steps and the control mechanisms. He emphasized the need for investigating the enzymes which catalyse each step, and he brought to the attention of the meeting other conditions, such as environmental and genetical factors, which could affect biosynthesis.

The first paper on a particular group of plant constit. uents was presented by Prof. T. W. Goodwin (University of Aberystwyth), who gave an account of the formation of terpenoids, compounds which appear to play a key part in chloroplast development. When etiolated seedlings are illuminated there is a massive synthesis of terpenoids as the functional chloroplasts are produced. During the greening period, carbon dioxide but not mevalonate is incorporated into the chloroplast terpenoids, but the reverse is true for the related sterols which are also present. It is believed that the biosynthesis of terpenoids in developing seedlings is regulated by enzymo segregation and specific membrane permeability. A second paper concerning the detailed steps involved in the elaboration of carotenoids, starting from acetate, was also presented by Prof. Goodwin.
An account of the amino-acids found in plants was given by Dr. L. Fowden (University College, London). In his lecture he concentrated on the large number of nonproteinogenic derivatives of which at least two groups are recognized. First, those which are intermediates in the formation of the twenty protein amino-acids and also those which arise by the non-specific action of enzymes normally associated with the metabolism of the proteinogenic compounds. The role of most of them is unknown.

Studies with micro-organisms have provided evidence that DNA controls the synthesis of proteins. This evidence was reviewed together with the mechanism of amino-acid polymorization by Dr. D. Boulter (University of Liverpool). $\mathrm{H}_{\theta}$ also described the similar findings made with higher plants and commented on the work being carried out at the University of Liverpool on protein synthesis in developing seeds.

The vast field of carbohydrate biochemistry was also reviewed (Dr. J. S. D. Bacon, Macaulay Institute, Aberdeen) mainly with reference to simple sugar phosphates and the more complex nucleotides derived from them. The synthesis of insoluble polymeric carbohydrates still remains something of a mystery. For example, although cellulose is the most abundant plant polysaccharide, we know very little about the formation of this material. Recent investigations by Hassid et al. ${ }^{1}$ at Berkeley have implicated guanosine diphosphate glucose, and Prof. R. D. Preston (University of Leeds) was able to tell the Group of other interesting observations, of a biophysical nature, which help in the general understanding of this problem. He discussed the arrangement of collulose microfibrils in the cell-walls of seaweeds and showed electron micrographs of spherical granules which are apparently involved in the synthesis and orientation of the polymer strands.

An equally complex problem, which was summarized by Dr. F. A. Isherwood (Low Temperature Research Station, Cambridge), is the structure and biogenesis of lignin. Progress in this field has slowed down considerably during the past few years and the molecule still refuses to give up all its secrets. There is little doubt, however, that the aromatic rings in this polyphenol are derived from shikimic acid and it is probable that the 